\title{
Crecimiento de grano tridimensional con partículas móviles usando método de Monte Carlo
}

\section{Three-dimensional grain growth with mobile particles using Monte Carlo method}

Pastor Ignacio Achával ${ }^{1}$, Carlos Leonardo Di Prinzio ${ }^{1,2}$

\author{
${ }^{1}$ Facultad de Matemática, Astronomía y Física -FaMAF/UNC- Medina Allende s/n, Ciudad Universitaria CP: \\ X5016LAE, Córdoba, Córdoba, Argentina. \\ e-mail:pachaval@famaf.unc.edu.ar; diprinzio@famaf.unc.edu.ar \\ ${ }^{2}$ Instituto de Física Enrique Gaviola (IFEG-CONICET), Medina Allende s/n, Ciudad Universitaria CP: X5016LAE, \\ Córdoba, Córdoba, Argentina. \\ e-mail: sec_if@famaf.unc.edu.ar
}

\section{RESUMEN}

En este trabajo se presenta un modelo de simulación de crecimiento de grano con partículas móviles en tres dimensiones usando el método Monte Carlo. Se estudió el efecto de la concentración y el tamaño de las partículas sobre el tamaño de grano. En general, se pudo observar la migración de las partículas hacia los bordes de grano y el frenado que las mismas producen sobre el movimiento de los límites de grano. También se observó a tiempos largos un efecto de liberación de los bordes de grano respecto a las partículas.

Se determinó el radio crítico donde los granos se frenan para cada concentración y cada tamaño de partícula. Finalmente, se presenta la relación entre el radio crítico, el tamaño de las partículas y concentración de las mismas. Se concluyó que el radio crítico es en general igual al radio de la esfera equivalente al volumen entre las partículas.

Palabras clave: Monte Carlo, Crecimiento de Grano, Partículas móviles, Partículas de Segunda Fase, Efecto Zener.

\begin{abstract}
In this paper we present a simulation model of grain growth with moving particles in three dimensions using the Monte Carlo method. The effect of concentration and particle size on grain size was studied. In general, it was possible to observe the migration of the particles towards the grain boundaries and the braking that they produce on the movement of the grain boundaries. An effect of releasing the grain boundaries from the particles was also observed at long times. The critical radius, where the grains were braked for each concentration and particle size, was determined. Finally, the relation between the critical radius, the particle size and the concentration of the particles is presented. It was concluded that the critical radius is generally equal to the radius of the sphere equivalent to the volume between the particles.
\end{abstract}

Keywords: Monte Carlo, Grain Growth, Moving Particles, Second Phase Particles, Zener Effect.

\section{INTRODUCCIÓN}

El método de Monte Carlo (MC) ha sido utilizado para simular numéricamente el crecimiento de grano (CG) en muestras policristalinas bidimensionales (2D) [1,2]. YU y ESCHE [3] presentaron un análisis cinético y topológico del MC en CG en muestras policristalinas en tres dimensiones (3D) sin partículas.

Con el mismo método se estudiaron efectos de las impurezas inmóviles [4] y móviles [5] sobre muestras 2D y efectos de las impurezas inmóviles sobre muestras policristalinas tridimensionales [6].

El CG con partículas de segunda fase dispersa es de interés en glaciología. El CG en muestras de hielo polar y su relación con los contaminantes se encuentra ligado al clima del pasado y su investigación es relevante hoy día para conocer el comportamiento del clima [7]. En los hielo polares se encuentran, en 
general, partículas que pueden moverse o no con el borde de grano (BG) y el efecto de estas partículas en el CG todavía es tema de discusión [8]. Las simulaciones numéricas pueden ayudar a entender este fenómeno.

DI PRINZIO et al. [9] encontraron que las partículas inmóviles frenan el crecimiento de grano hasta detenerlo y que en 3D se cumple la ley de Zener:

$$
R_{c}=\frac{4}{3} \frac{r}{f}
$$

donde $R_{c}$ es el radio máximo que alcanzan los granos debido a la influencia de las partículas, $f$ la fracción volumétrica de partículas y $r$ el radio de las partículas. Lo relevante de este trabajo es que se hicieron simulaciones con distintos tamaños de partículas. Los granos son identificados con un radio medio $R$ que representa el radio de una esfera cuyo volumen es equivalente al volumen medio de los mismos.

El radio medio de los granos, $R$, ha sido representado por la ecuación:

$$
\frac{d R}{d t}=k\left(\frac{1}{R}-\frac{1}{R_{c}}\right)
$$

donde $k$ es una constante cinética del CG.

La integración de la ecuación anterior da por resultado:

$$
k\left(t-t_{0}\right)=-R_{c}\left(R_{c} \log \left(\frac{R-R_{c}}{R_{0}-R_{c}}\right)+\left(R-R_{0}\right)\right)
$$

donde $R_{0}$ es el radio al tiempo inicial $t_{0}$ y se encuentra citada por GREY y HIGGINS [10].

Los datos obtenidos por DI PRINZIO et al. [11] fueron ajustados por la ecuación anterior a fin de encontrar el valor de $R_{c}$.

Asimismo, el trabajo de HASSOLD y SROLOVITZ [5] se basó en simulaciones con Monte Carlo del CG en 2D con partículas móviles cúbicas unitarias. En este importante trabajo se encontró que los bordes de grano (BGs) migraban inicialmente siguiendo la ecuación (3). A partir de un cierto tiempo $t_{\mathrm{c}}$ los granos adquirían en promedio un tamaño de grano medio caracterizado por $R_{\mathrm{c}} \mathrm{y}$ se frenaba notablemente el CG. Según los autores el tiempo $t_{\mathrm{c}}$ seguía la ecuación:

$$
t_{c}=\frac{R_{c}^{2}}{2 k_{c}}
$$

donde $k_{\mathrm{c}}$ es una constante cinética intrínseca del CG (ecuación (2)).

Sin embargo, el CG se estancaba fuertemente hasta un cierto tiempo $t_{h}$ donde los BGs se liberaban de las partículas y se aceleraban. El tiempo $t_{h}$ estaba dado por la ecuación:

$$
t_{h}=\frac{R_{c}^{2}}{2 k_{l}}
$$

donde $k_{l}$ es la constante cinética intrínseca del CG (ecuación (2)) que según los autores es: $k_{l}=\beta / \mathrm{f}$ y $\beta$ es un parámetro físico que depende de la interacción de las partículas con el BG. Debemos decir que las ecuaciones (4) y (5) propuestas por HASSOLD y SROLOVITZ [5] son válidas cuando se aproxima $R_{c}$ a tiempo cortos por:

$$
R_{c}^{2} \approx 2 k_{c} t
$$

Esta aproximación resulta poco creíble por los resultados hallados en otros trabajos [1-6].

El trabajo de HASSOLD y SROLOVITZ [5] es, sin embargo, muy importante en relación al CG con partículas móviles, pero debemos destacar que los resultados con simulaciones de MC del CG en 3D con partículas móviles son muy escasos y no han sido hechos para diferentes tamaños de partículas [6, 9, 11-17].

La motivación de este trabajo es presentar por primera vez un estudio de CG utilizando una nobel adaptación del método de Monte Carlo en 3D [1-3, 9] y ver los efectos del tamaño de las partículas móviles y su concentración sobre el CG. Posiblemente estos resultados puedan contribuir a la interpretación y esclarecimiento de fenómenos físicos en muchos materiales y en particular en el hielo polar.

\subsection{Algoritmo de Cálculo}

La simulación del CG con MC en muestras en 3D usa los mismos procedimientos que se emplean en muestras en 2D [1,2]. 
Inicialmente se crea una red 3D con $Q$ sitios $\left(\mathrm{N}_{\mathrm{x}} \times \mathrm{N}_{\mathrm{y}} \times \mathrm{N}_{\mathrm{z}}=\mathrm{Q}\right)$ donde cada sitio $i$ representa un grano con una orientación única $S_{i}>0$. En este modelo el policristal inicial tiene todos los granos con $S_{i}=1 \ldots Q$ diferentes. El algoritmo diferencia entre sitios pertenecientes a un cristal o grano (S $\left.>0\right)$ y sitios pertenecientes a una partícula $(\mathrm{S}=0)$.

Para sitios con $\mathrm{S}>0$ el algoritmo sigue los siguientes pasos:

a) La energía total del policristal $W$ está dada por:

$$
W=\frac{1}{2} \sum_{i=1}^{Q} \sum_{j=1}^{V} J\left(1-\delta_{S i S j}\right)
$$

Con $S_{i}$ y $S_{j}$ orientaciones de los sitios de la red y pertenecientes a un grano $i$ y $j$ respectivamente, $J$ energía de interacción entre sitios, $Q$ número de sitios de red, $V$ número de vecinos al sitio $i$, y $\delta$ función delta de Kronecker. En este trabajo se tomó $V=26$ vecinos y la red de puntos fue de $Q=100 \times 100 \times 100$ pixel $^{3}$ (nombre utilizado para referenciar una unidad en la muestra)

b) En forma aleatoria se elige un sitio de la red perteneciente a un grano denominado $i$ con una orientación $S_{i}$. Mediante la ecuación (7) se calcula la energía alrededor del sitio $i$ :

$$
W_{i}^{i n}=\sum_{j=1}^{V} J\left(1-\delta_{S i S j}\right)
$$

donde el supra índice in significa etapa inicial.

c) Luego se remplaza la orientación del sitio $i, S_{i}$, por una orientación $S_{j}$ del sitio $j$ obtenida aleatoriamente de sus vecinos que pertenecen a un grano.

d) Se calcula nuevamente la energía del sitio $i, W_{i}^{f i}$, donde el supra índice $f i$ significa final.

e) Luego se calcula la diferencia de energías:

$$
\Delta W_{i}=W_{i}^{f i}-W_{i}^{i n}
$$

f) Si la ecuación (9) resulta negativa o nula el cambio se produce permanentemente y si es positiva se calcula una probabilidad $P$ dada por:

$$
P=e^{\frac{-\Delta W_{i}}{k T}}
$$

donde $k$ es la constante de Boltzman y $T$ es la temperatura del policristal. Para permitir que el sistema produzca cambios por activación térmica, se elige un número aleatorio $M$ entre 0 y 1 , y se compara con $P$. Si $P$ es más grande que $M$ entonces se hace el cambio de $S_{i}$ por el $S_{j}$, en caso contrario no. En todas las simulaciones se consideró $k T \approx 0,5 \mathrm{~J}$.

Las reglas que rigen el movimiento de las partículas móviles son los siguientes:

a) Al igual que ANDERSON et al. [6] las partículas móviles son representadas con la orientación $S=$ 0 y se mantiene fija su concentración a lo largo de toda la simulación. Las posiciones iniciales de las mismas se establecen distribuyéndolas uniformemente en la muestra. Las partículas estudiadas estaban formadas por un conjunto de sitio de la muestra con $S=0$ constituyendo cubos de lados 1,2 o 3 pixels según fuera el tamaño de las partículas utilizadas en la simulación.

b) Se elige un sitio w al azar de la muestra y se verifica que el mismo tenga $S=0$, o sea, que pertenezca a una partícula o a parte de ella.

c) Para mover una partícula debíamos primero asegurarnos que el sitio elegido al azar pertenecía al centro geométrico de la partícula. También era necesario saber si la partícula estaba dentro de un grano $(\mathrm{G})$, en un borde de grano (BG) o cerca del límite de la muestra. La partícula es movida solamente si el sitio elegido w es centro de una partícula y la misma está en el G o en el BG. Si la partícula está en el G se elige un vecino del sitio $\mathrm{w}$ al azar y se mueve a esa posición el centro de la partícula (eso significa que se mueven simultáneamente todos los sitios con $S=0$ que forman la partícula cúbica). Si la partícula está en el BG, se elige al azar un vecino del sitio w y se mueve a esa posición el centro de la partícula siempre que la ecuación (9) de cero o un número negativo. Si la ecuación (9) da un número positivo se mueve la partícula si y sólo si la probabilidad $P$ de la ecuación (9) es más grande que el número aleatorio $M$ (definido anteriormente). 
d) Debemos aclarar que si la partícula está en el G y el sitio vecino elegido aleatoriamente produce un movimiento de la partícula en dirección al límite de la muestra se prohíbe ese estado y se elige un nuevo sitio vecino de manera aleatoria.

\section{MATERIALES Y MÉTODOS}

Se utilizó el programa de crecimiento de Grano en 3D, en muestras de $100 \times 100 \times 100$ pixel $^{3}$ con y sin partículas móviles y con energía de BG uniforme.

Se simularon policristales cúbicos sin partículas y con 1\%, 2\%, 3\%, 4\%, 5\% y $10 \%$ de partículas móviles de tres tamaños diferentes $\left(1\right.$ pixel $^{3}, 27$ pixel $^{3}$ y 125 pixel $\left.^{3}\right)$. Las simulaciones se realizaron partiendo de una muestra con granos de radio inicial de 1 pixel $^{3}$ y se hicieron cerca de 60.000 pasos de computadora (MCS) equivalentes a 3 días de simulación.

En cada paso se fue registrando el tamaño medio $R$ de los granos de la muestra.
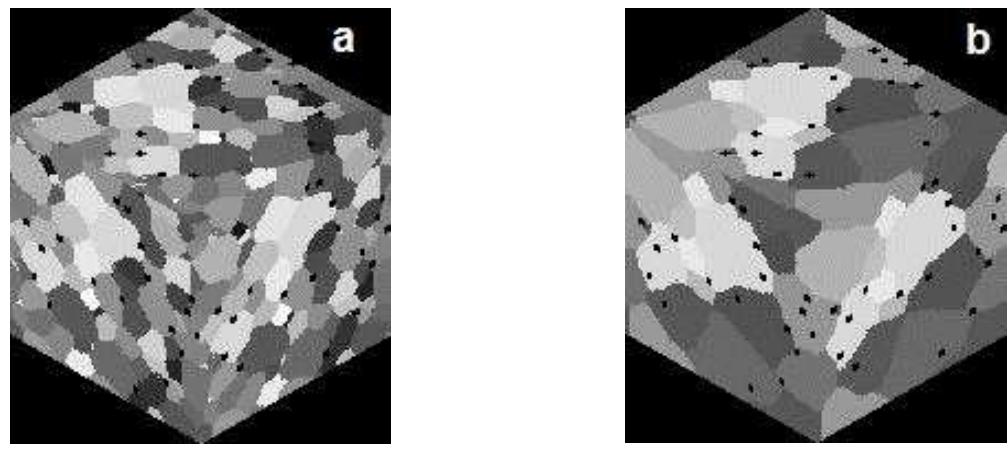

Figura 1: Policristal con partículas cúbicas de lados de 3 pixels y concentración 4\%: a) $t=1000$ MCS y b) $t=10000$ MCS.

En la figura 1 se presenta una muestra de un policristal en 3D usado en la simulación a los 1000 MCS y a los 10000 MCS. Tiene partículas cúbicas de lado de 3 pixels y una concentración volumétrica de las mismas de $4 \%$. Las partículas son representadas por puntos negros y los granos por distintas tonalidades de grises.

\section{RESULTADOS DE LAS SIMULACIONES:}

En la figura 1 podemos ver como las partículas móviles se van ubicando en el BG de la muestra mientras el tamaño medio de los granos crece.

En la figura 2 se muestra $R$ en función de $t$ en MCS para muestras con partículas cúbicas de lado 1 pixel con concentraciones de $0 \%, 1 \%, 3 \%, 5 \%$ y $10 \%$. Podemos ver que, en general, para todas las concentraciones, el CG prácticamente se detiene hasta por lo menos los 50.000 MCS que duraron las simulaciones.

En la figura 3 se presentan los valores de $R$ en función de MCS hasta los 10.000 MCS para muestras con partículas cúbicas de lado 3 pixels con concentraciones de 0\%, 1\%, 2\%, 3\%, 4\%, 5\% y 10\%. Podemos ver que para las muestras con 4\%, 5\% y $10 \%$ los CGs presentan un frenado significativo en los primeros 10.000 MCS hasta casi impedir el CG, sin embargo las muestras con 1\%, $2 \%$ y $3 \%$ sufren un leve frenado en el CG.

En la figura 4 se presenta $R$ en función de MCS para muestras con partículas cúbicas de lado 5 pixels con concentraciones de $0 \%, 1 \%, 2 \%, 3 \%, 4 \%, 5 \%$ y $10 \%$. Podemos ver que las muestras con concentraciones de $2 \%, 3 \%, 4 \% 5 \%$ y $10 \%$ presentan un frenado importante. En las muestras de $2 \%, 3 \%$ y $4 \%$ la detención del CG es temporal observándose una posterior aceleración del CG, mientras que en las muestras del 5\% y $10 \%$ el detenimiento del CG es prácticamente total hasta los 60.000 MCS que duraron las simulaciones.

En general se observan dos tipos de muestras:

1) Muestras con frenado (MCF): son aquellas muestras donde la partículas están a poca distancia y el CG prácticamente se detiene en un tamaño $R_{c}$ a un tiempo $t_{c}$.

2) Muestras sin Frenado (MSF): Aquellas muestras donde las partículas están bastante separadas entre sí y el CG se va frenando levemente a un tiempo $t_{c}$ para un radio $R_{c}$ pero no se detiene nunca. 
También se pudo constatar que, para muestras con igual concentración $f$, el valor de $R_{c}$ es más grande en muestras con partículas más grandes.

En la figura 5 se presenta el tamaño de grano medio $R$ en función del MCS para una muestra con partículas cúbicas de lado 3 pixels y una concentración volumétrica de $4 \%$. Se puede ver que $R$ es ajustado muy bien por la ecuación (4) que representa el CG con frenado según GREY y HIGGINS [11]. Podemos obtener de este ajuste el radio $R_{c}$.

Por otro lado, el tiempo $t_{c}$ se tomó como el tiempo donde el radio $R$ de los granos comenzó a variar menos del $5 \%$.

Para cada policristal presentado en las figuras 2, 3 y 4 con distinto tamaño de partículas y concentración se extrajo $R_{c}$ y $t_{c}$, al igual que lo hizo HASSOLD y SROLOVITZ [5], usando el ajuste de acuerdo a la ecuación (4). Se ajustaron los datos desde la parte inicial hasta donde el tamaño de grano variaba sólo un 5\%. HASSOLD y SROLOVITZ [5] sólo usaron un tamaño de partícula pero pudieron ver que algunas muestras se comportaban como MCF y otras como MSF.

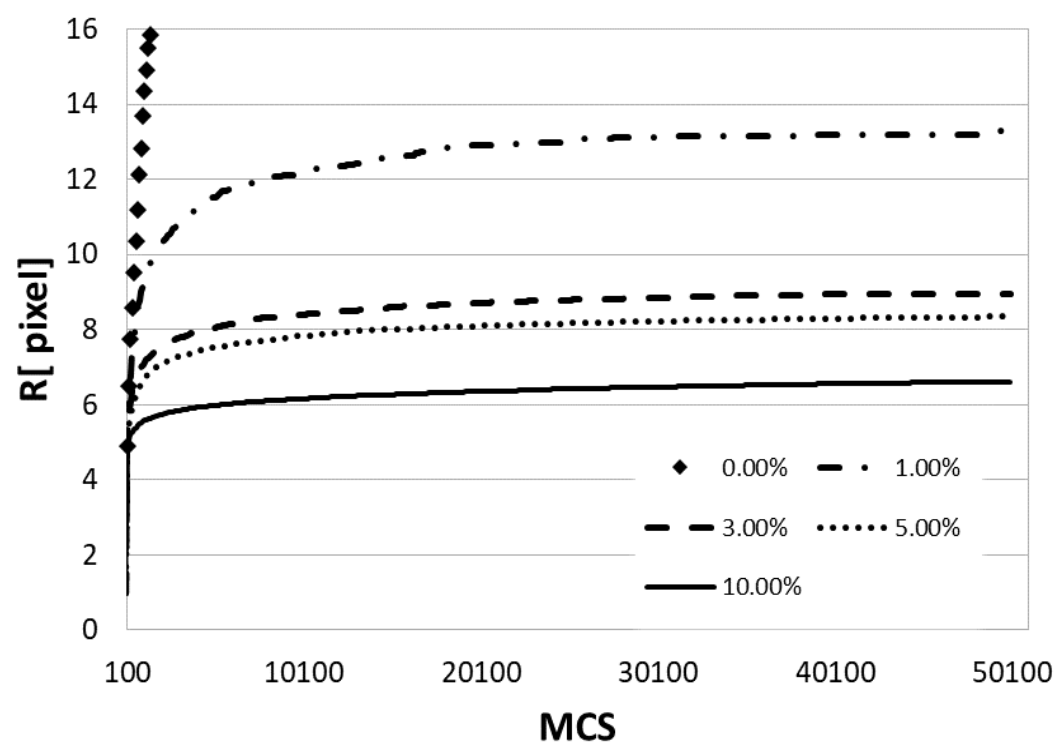

Figura 2: $R$ en función de MCS para muestras con partículas cúbicas de lado 1 pixel y concentraciones de partículas de $0 \%, 1 \%, 3 \%, 5 \%$ y $10 \%$.

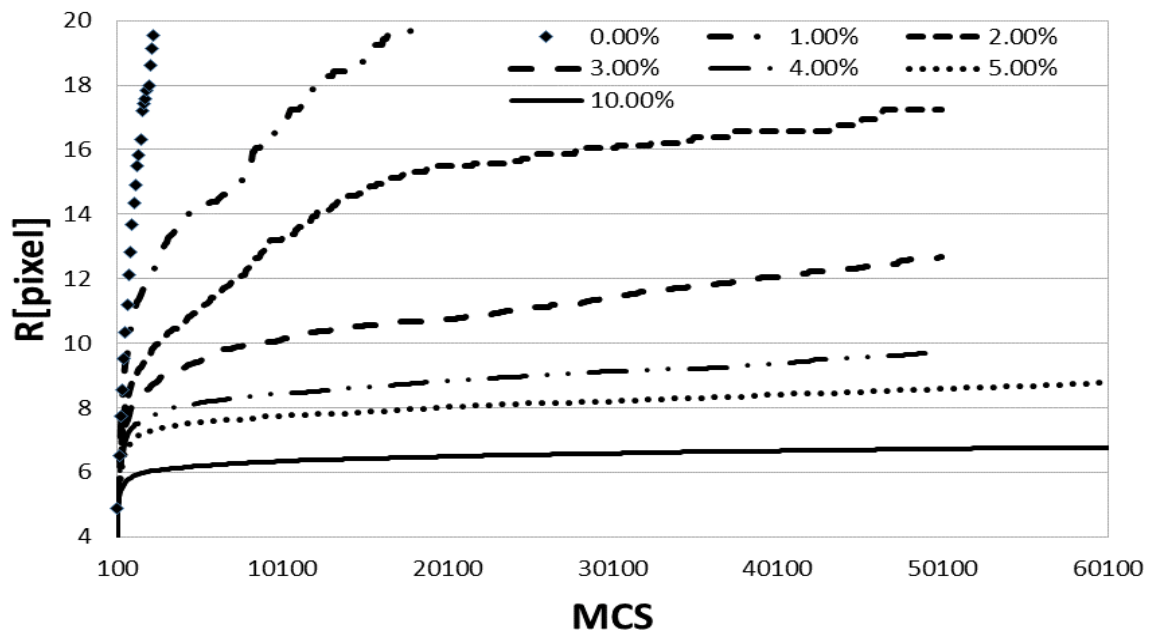

Figura 3: $R$ en función de MCS para muestras con partículas cúbicas de lado 3 pixels y concentraciones de partículas de $0 \%, 1 \%, 2 \%, 3 \%, 4 \%, 5 \%$ y $10 \%$. 


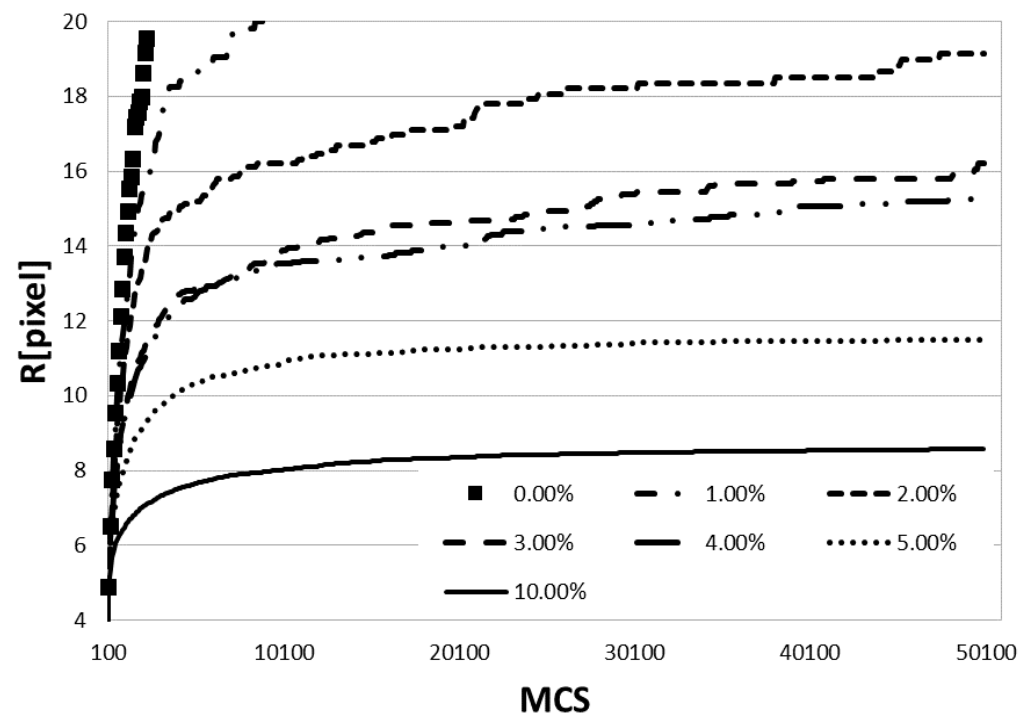

Figura 4: $R$ en función de MCS para muestras con partículas cúbicas de lado 5 pixels y concentraciones de partículas de $0 \%, 1 \%, 2 \%, 3 \%, 4 \%, 5 \%$ y $10 \%$.

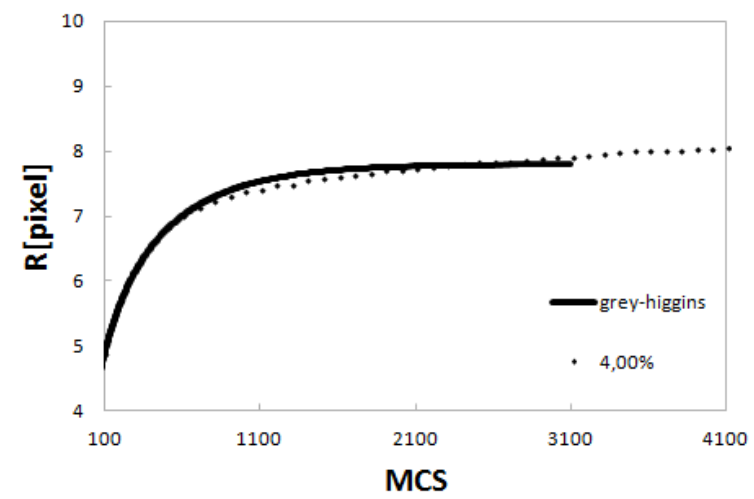

Figura 5: $R$ en función de MCS para muestras con 4\% de partículas cúbicas de lado 3 pixels.

Podemos entonces calcular $R_{c} / r$ en función de $f$ (figura 6) donde $r$ es el radio equivalente de una esfera con el mismo volumen que la partícula. Podemos ver que para las partículas mas grandes $R_{c} / r$ en función de $f$ sigue la siguiente relación:

$$
\frac{R_{c}}{r}=a f^{b}
$$

donde $b=-0,38 \pm 0,04$ y $a=1,2 \pm 0,2$.

Los datos de $R_{c} / r$ vs. $f$ para las partículas más chicas tienen la misma pendiente y se nota que los valores de $R_{c}$ son grandes para que los valores de $R_{c} / r$ coincidan con los datos de las partículas más grandes. Eso podría estar relacionado con el unpining (o desprendimiento) de las partículas del BG que ya había observado DI PRINZIO et al. [9].

Podemos notar además que si se calcula $R_{l}$ que es el radio del volumen equivalente esférico entre las partículas con radio $r$ y concentración $f$ se tiene la siguiente relación:

$$
\frac{R_{l}}{r}=a f^{b} \text { donde } a=1 \text { y } b=-1 / 3
$$

Podemos ver que el exponente $b$ y el factor pre-exponencial de la simulación (ecuación (11)) coinciden muy bien con los valores deducidos de la teoría. 


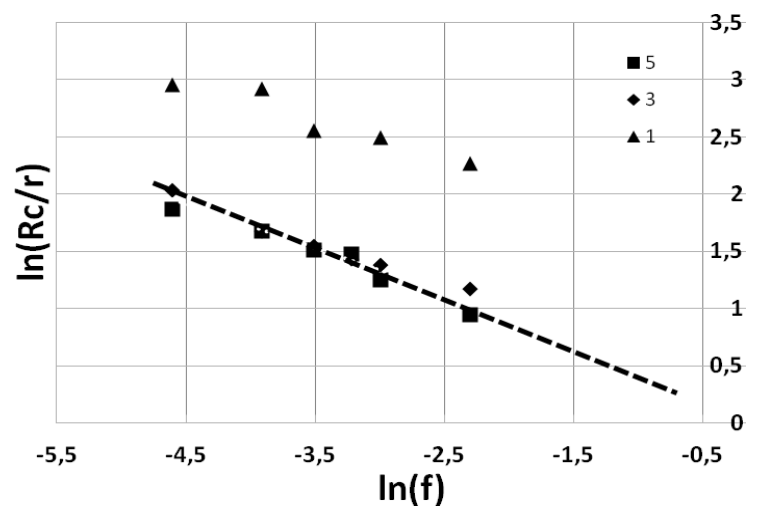

Figura 6: $R_{c} / r$ en función de $f$ para las muestras simuladas con partículas de lado 1,3 y 5 pixels a diferentes concentraciones.

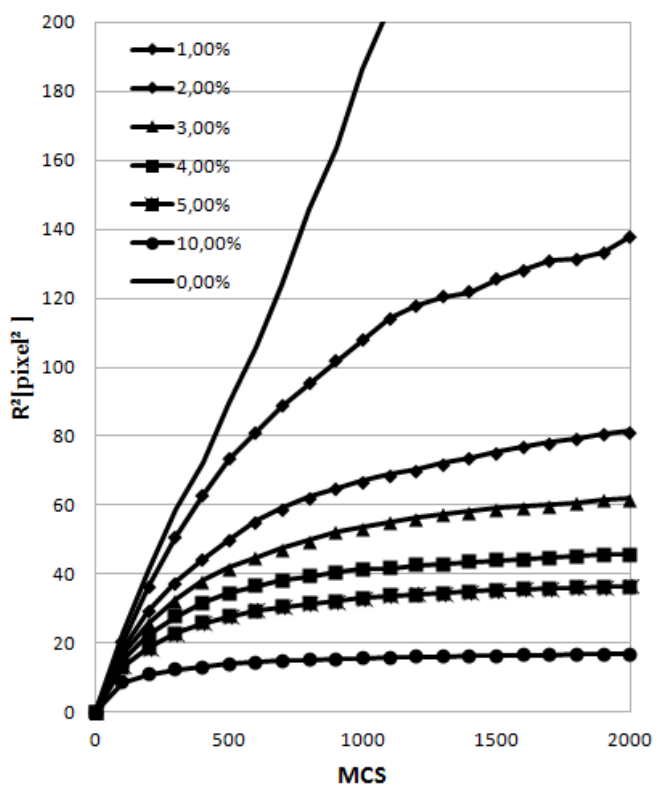

Figura 7: $R_{c}{ }^{2}$ en función de MCS para las muestras simuladas con partículas de lado 3 pixels a diferentes concentraciones.

Podemos, entonces, decir que el radio $R_{c}$ es prácticamente el radio $R_{l}$ del volumen libre que existe entre las partículas.

Se investigó qué ocurre con el tiempo $t_{c}$ en nuestras simulaciones. Pudimos ver que tc no se comporta como la ecuación (4). Creemos que esa discrepancia se puede deber a que el valor de $\mathrm{R}^{2}$ no guarda una la relación lineal con MSC como se propone en la ecuación (6) dada por HASSOLD y SROLOVITZ [5].A modo de ejemplo, en la figura 7 se muestra $R^{2}$ vs. MCS para CG con partículas cúbicas de lado 3 pixel a diferentes concentraciones y donde la relación entre ambas variables no es lineal, salvo para el caso de muestras libres de partículas. Por lo tanto no se pueden aplicar las ecuaciones (4) en nuestros datos como lo hicieron HASSOLD y SROLOVITZ [5] para hallar el tiempo $t_{c}$.

\section{CONCLUSIONES}

En este trabajo se presentaron resultados simulados de CG con partículas cúbicas móviles de lado 1,3 y 5 pixels a diferentes concentraciones en el rango de $0 \%$ a $10 \%$.

Los resultados muestran que:

1) El crecimiento de los granos con partículas móviles de distinto tamaño se detiene para un cierto tamaño de los granos (al que denominamos “crítico") parcialmente para muestras del tipo MSF y totalmente para muestras del tipo MCF. 
2) El valor de $R_{c}$ en el CG depende de la fracción de partículas móviles como una potencia de $-1 / 3$ y del tamaño de las mismas, siguiendo la ecuación (11).

3) Ese detenimiento parcial o total se produce cuando el diámetro del volumen esférico equivalente de los cristales es del orden de la distancia entre las partículas.

4) Queda para futuros trabajo encontrar que combinación de variables produce una muestra MCF o una muestra MSF.

\section{AGRADECIMIENTOS}

Este trabajo fue posible gracias a la colaboración de José Barcelona y del apoyo económico de la secretaria de Ciencia y técnica de la UNC.

\section{BIBLIOGRAFÍA}

[1] ANDERSON, M.P., SROLOVITZ, D.J., GREST, G S. et al., "Computer simulation of grain growth - I. Kinetics”, Acta Metallurgica, v. 32, n. 5, pp. 783-791, May. 1984.

[2] SROLOVITZ, D.J., ANDERSON, M.P., SAHNI, P.S. et al., "Computer simulation of grain growth - II. Grain size distribution, topology, and local dynamics", Acta Metallurgica, v. 32, n. 5, pp. 793-802, May. 1984.

[3] YU, Q., ESCHE, S.K., "Three-dimensional grain growth modeling with a Monte Carlo algorithm", Materials Letters, v. 57, n. 30, pp. 4622-4626, Dec. 2003.

[4] GAO, J., THOMPSON, R.G., PATTERSON, R., "Computer simulation of grain growth with second phase particle pinning”, Acta Materialia, v. 45, n. 9, pp. 3653-3658, Sept. 1997.

[5] HASSOLD, G.N., SROLOVITZ, D.J., "Computer Simulation of Grain Growth with mobile particles", Scripta Metallurgica et Materialia, v. 32, n. 10, pp. 1541-1547, May. 1995.

[6] ANDERSON, M.P., GREST, G.S., DOHERTY, R.D., et al., "Inhibition of grain growth by second phase particles: Three dimensional Monte Carlo computer simulations”, Scripta Metallurgica, v. 23, n. 5, pp. 753758, May. 1989.

[7] GOW, A.J., MEESE, D., "Physical properties, crystalline textures and c-axis fabrics of the Siple Dome (Antarctica) ice core", Journal of Glaciology, v. 53, n. 183, pp. 573-584, Dec. 2007.

[8] DI PRINZIO, C.L., WILEN, L.A., ALLEY, R.B., et al., "Fabric and texture at Siple Dome, Antarctica", Journal of Glaciology, v. 51, n. 173, pp. 281-290, Mar. 2005.

[9] DI PRINZIO, C.L., DRUETTA, E., NASELLO, O.B., "More about Zener drag studies with Monte Carlo simulations", Modelling and Simulation in Materials Science and Engineering, v. 21, n. 2, pp. 025007, Jan. 2013.

[10] GREY, E.A., HIGGINS, G.T., “A velocity independent drag during grain boundary migration”, Scripta Metallurgica, v. 6, n. 3, pp. 253-258, Mar. 1972.

[11] NOVIKOV, V.Y., "Grain growth controlled by mobile particles on grain boundaries", Scripta Materialia, v. 55, n. 3, pp. 243-246, Aug. 2006.

[12] MALLICK, A., "Effect of second phase mobile particles on polycrystalline grain growth: A phase-field approach”, Computational Materials Science, v. 67, pp. 27-34, Feb. 2013.

[13] NOVIKOV, V.Y., "Grain growth jointly affected by immobile and mobile particles". Materials Letters, v. 178, pp. 276-279, Sept. 2016.

[14] NOVIKOV, V.Y., "On grain growth in the presence of mobile particles“, Acta Materialia, v. 58, n. 9, pp. 3326-3331, May. 2010.

[15] NOVIKOV, V.Y., "Abnormal grain growth in thin films not caused by decreased energy of their free surface”, Materials Letters, v. 65, n. 17-18, pp. 2618-2620, Sept. 2011.

[16] NOVIKOV, V.Y., "Grain growth suppression in nanocrystalline materials", Materials Letters, v. 100, pp. 271-273, Jun. 2013.

[17] AHMED, K., TONKS, M., ZHANG, Y., BINER, B., EL-AZAB, A., "Particle-grain boundary interactions: A phase field study”, Computational Materials Science, v. 134, pp. 25-37, Jun. 2017. 